\title{
A Novel Dynamic Absorber with Variable Frequency and Damping
}

\author{
Tian Wang $\mathbb{D}^{1,2,3}$ Ruilan Tian $\mathbb{D}^{1,2,3}$ Xinwei Yang ${ }^{1},{ }^{4}$ Ziwen Zhang $\mathbb{D}^{1,2,3}$ \\ and Xiaolong Zhang $\mathbb{D}^{1,2,3}$ \\ ${ }^{1}$ State Key Laboratory of Mechanical Behavior and System Safety of Traffic Engineering Structures, \\ Shijiazhuang Tiedao University, Shijiazhuang 050043, China \\ ${ }^{2}$ Department of Engineering Mechanics, Shijiazhuang Tiedao University, Shijiazhuang 050043, China \\ ${ }^{3}$ Key Laboratory of Smart Materials and Structures Mechanics, Shijiazhuang Tiedao University, Shijiazhuang 050043, China \\ ${ }^{4}$ Shijiazhuang Institute of Railway Technology, Shijiazhuang 050041, China
}

Correspondence should be addressed to Ruilan Tian; tianrl@stdu.edu.cn

Received 24 September 2020; Revised 19 January 2021; Accepted 1 February 2021; Published 12 February 2021

Academic Editor: Matteo Filippi

Copyright (C) 2021 Tian Wang et al. This is an open access article distributed under the Creative Commons Attribution License, which permits unrestricted use, distribution, and reproduction in any medium, provided the original work is properly cited.

\begin{abstract}
Smoothness and discontinuous (SD) oscillator is a nonlinear oscillator with the variable frequency, whose frequency can be varied with the smoothing parameter. However, how to adjust the smoothing parameter has not been solved in the actual device. In this paper, the shape memory alloy (SMA) is introduced into the SD oscillator to form the SMA-SD oscillator to adjust the smoothing parameters. Combining the SMA-SD oscillator with MRF, a nonlinear dynamic vibration absorber (DVA) with variable frequency and damping is designed. The structure and control principle of the designed DVA is studied to achieve the two variable characteristics simultaneously by adjusting the current intensity. Numerical results on a two-degree-of-freedom coupled system show that the proposed DVA can adapt to different working conditions only by adjusting the current intensity.
\end{abstract}

\section{Introduction}

Vibration widely exists in civil engineering and mechanical industry. Requirements of dynamic vibration absorbers (DVAs) are different for variable environments. Therefore, it is an urgent problem to construct a DVA with the variable frequency which can meet the changeable working conditions. Smooth and discontinuous (SD) oscillator was a nonlinear system proposed by Cao [1] in 2006. Due to its geometric nonlinearity, the restoring force yielded the irrational nonlinear form, which made its frequency vary depending on the smoothness parameter $\alpha$. In recent years, the applications of SD oscillator for the vibration reduction have been studied [2,3], which showed that the desired effect of the vibration reduction can be achieved by adjusting the smooth parameter $\alpha$. However, in these papers, how to change $\alpha$ to realize the frequency conversion is barely mentioned.

In the SD oscillator system, the smoothing parameter $\alpha$ is related to the original length $l$ of the springs. If $l$ changes, $\alpha$ will be different accordingly. In this paper, the shape memory property of the shape memory alloy (SMA) will be used to adjust the smooth parameters of the SD oscillator. SMA is a functional material with sensing and driving characteristics [4]. The springs made of SMA can be extended or shortened by controlling the temperature. Therefore, the length of SMA springs can be controlled by using the thermal effect of the current, which will change the geometric nonlinear structure of the SD oscillator to achieve the variable frequency.

To a certain extent, a nonlinear structure such as the SD oscillator can effectively adjust the frequency of the system and reduce the vibration. For example, Ding [5] built a nonlinear isolation system with the quasizero stiffness, which had three springs for the transverse vibration of prepressure beams. Jing et al. [6] investigated a passive bioinspired limb-like structure (LLS). Hence, the dynamic absorber with the variable frequency was beneficially adaptable to the changeable working conditions.

For the nonlinear damping, magnetorheological fluid (MRF) was a controllable fluid discovered by Jacob [7] in the late 1940s, whose rheological behavior can change 
dramatically under the action of the magnetic field. Based on the advantages of rapid response, low energy consumption, and large shear stress, MRF has been very useful for vibration control. More critically, it can be controlled by using the magetic effect of th current.

The main purpose of this paper is to solve the problem of the unadjustable smoothness parameter in the SD oscillator system. Combining the SMA-SD oscillator and magnetorheological fluid (MRF), a nonlinear dynamic vibration absorber (DVA) with variable frequency and damping will be constructed, whose frequency and damping can be controlled simultaneously by adjusting the current intensity.

The main structure of this paper is as follows. In Section 2, the implementation of the variable frequency for the SD oscillator system is constructed. In the next section, the structure and principle of the nonlinear DVA with variable frequency and damping are designed. Furthermore, the expression of the damping force provided by MRF is given. In Section 4, the designed nonlinear DVA is applied to a variable-section beam to discuss the response of the system under an infinite series of moving loads, demonstrating the damping performance and potential for suppressing broadband vibration of the designed DVA.

\section{Variable-Frequency Implementation for SD Oscillator System}

2.1. Relationship between the Elongation of SMA Spring and Temperature. For normal cylindrical helical springs subjected to tension or compression, the load force acts on the axis of the springs. The restoring force of the spring is [8]

$$
F=\frac{G d^{4}}{8 N D^{3}} \delta
$$

where $F, G, N, D, d$, and $\delta$ are the external force, the shear modulus, the number of the spring coils, the diameter of the spring coils, the diameter of the spring wires, and the deformation of the spring under the load, respectively.

According to the theory of material mechanics, the shear strain $\gamma$ on the spring wire is [8]

$$
\gamma=\frac{\tau}{G}=\frac{8 F D}{\pi d^{3} G} .
$$

According to equations (1) and (2), the relationship between the spring's deformation $\delta$ and the shear strain $\gamma$ is

$$
\delta=\frac{\pi D^{2} N \gamma}{d} .
$$

For SMA material, there are four important transition temperatures: the martensite finish $\left(M_{f}\right)$, the martensite start $\left(M_{S}\right)$, the austenite end $\left(A_{f}\right)$, and the austenite start $\left(A_{S}\right)$ [9]. When the temperature changes, the properties of SMA springs will change and some deformation occurs. The relationship between the shear modulus and temperature is as follows [10]:

$$
G= \begin{cases}G_{M}, & T<M_{s}, \\ G_{T}, & M_{s} \leq T \leq A_{f}, \\ G_{A}, & T>A_{f},\end{cases}
$$

where $T, G_{M}$, and $G_{A}$ are the temperature, the shear modulus of SMA in the low-temperature martensite phase, and the shear modulus in the high-temperature austenite phase. $G_{T}=G_{M}+\left(G_{A}-G_{M} / 2\right)\left[1+\sin \omega\left(T-T_{M}\right)\right][10]$ is the shear modulus of the SMA between $M_{s}$ and $A_{f}$, where $T_{M}$ and $\omega$ are the parameters related to the temperature.

According to equations (1) and (3), under the external force $F$, we can conclude that

$$
\begin{aligned}
\delta_{L} G_{M} & =\delta_{T} G_{T}, \\
\frac{\gamma_{\max }}{\gamma_{T}} & =\frac{\delta_{L}}{\delta_{T}},
\end{aligned}
$$

where $\delta_{L}$ and $\gamma_{\max }$ are the displacement and the maximum shear strain when $T<M_{s}$ and $\delta_{T}$ and $\gamma_{T}$ are the displacement and the shear strain when $M_{s} \leq T \leq A_{f}$.

From equations (5) and (6), when $M_{s} \leq T \leq A_{f}$, the shear strain is $\gamma_{T}=\left(G_{M} \gamma_{\max } / G_{T}\right)$. Hence, according to equation (3), the length variation of the SMA spring is given by

$$
\begin{aligned}
l & =l_{0}+\Delta \delta=l_{0}+\frac{N \pi D^{2}}{d}\left(1-\frac{G_{M}}{G_{T}}\right) \gamma_{\max } \\
& =l_{0}+\frac{N \pi D^{2}}{d}\left(1-\frac{G_{M}}{G_{M}+\left(G_{A}-G_{M} / 2\right)\left[1+\sin \bar{\omega}\left(T-T_{M}\right)\right]}\right) \gamma_{\max },
\end{aligned}
$$

where $l_{0}$ is the initial length of the spring when the temperature is unchanged and $l$ is the length of the spring after the temperature changed. $\Delta \delta$ is the deformation of the spring when $M_{s} \leq T \leq A_{f}$. Clearly, the length of the SMA spring can be regulated by controlling the temperature.

2.2. Restoring Force and Stiffness of the SMA Spring. In fact, we can discuss the constitutive model of SMA from the microscopic point of view. The SMA constitutive model used in this paper is a stress-strain-temperature polynomial model proposed by Falk [11] in 1980. The free energy function of SMA depends on the strain $\varepsilon$ and the temperature $T$, without considering other variables. The stress $\sigma$, the strain $\mathcal{E}$, and the temperature $T$ of the model yield [11]

$$
\sigma=a_{1}\left(T-M_{f}\right) \varepsilon-a_{2} \varepsilon^{3}+a_{3} \varepsilon^{5}
$$

where $a_{1}, a_{2}, a_{3}$, and $M_{f}$ are positive. $A_{f}$ has the following form [12]:

$$
A_{f}=M_{f}+\frac{1}{4} \frac{a_{2}^{2}}{a_{1} a_{3}} .
$$


In the analysis of the spring deformation, there are shear stress $\bar{\tau}$ and shear strain $\bar{\gamma}$ [4]. From equations (8) and (9), introducing $\bar{\tau}=\sigma / \sqrt{3}$ and $\bar{\gamma}=\varepsilon / \sqrt{3}$, the relationship between $\bar{\tau}, \bar{\gamma}$, and the temperature $T$ is

$$
\bar{\tau}=a_{1}\left(T-M_{f}\right) \bar{\gamma}-3 a_{2} \bar{\gamma}^{3}+\frac{9}{4} \frac{a_{2}^{2}}{a_{1}\left(A_{f}-M_{f}\right)} \bar{\gamma}^{5} .
$$

Let $r$ be the radial coordinate of the SMA spring section. The relationship between the force $\bar{F}$ and the shear stress distribution on the spring wire section is

$$
\bar{F}=\frac{4 \pi}{D} \int_{0}^{d / 2} \bar{\tau} r^{2} \mathrm{~d} r
$$

where $D$ and $d$ represent the diameter of the spring and the diameter of the wire.

Suppose the shear strain is linearly distributed on the cross section [12]:

$$
\bar{\gamma}=\frac{2 r}{\pi D^{2} N} Y
$$

where $Y$ is the displacement of the spring.

The following equation can be obtained by equations (10)-(12):

$$
\bar{F}=\frac{4 \pi}{D} \int_{0}^{d / 2} \bar{\tau} r^{2} \mathrm{~d} r=\bar{F}_{1}+\bar{F}_{2}+\bar{F}_{3}
$$

where $\bar{F}_{1}=\left(a_{1}\left(T-M_{f}\right) Y d^{4} / 8 D^{3} N\right), \quad \bar{F}_{2}=-\left(\pi a_{2} Y^{3} d^{6} / 4\right.$ $\left.\left(\pi D^{2} N\right)^{3} D\right)$, and $\bar{F}_{3}=\left(9 \pi a_{2}^{2} Y^{5} d^{8} / 64 D a_{1}\left(\pi D^{2} N\right)^{5}\left(A_{f}-\right.\right.$ $\left.M_{f}\right)$ ).

From the expression of the spring recovery force, the stiffness of the SMA spring is as follows:

$$
\begin{aligned}
K & =\frac{\partial \bar{F}}{\partial Y} \\
& =\frac{a_{1}\left(T-M_{f}\right) d^{4}}{8 D^{3} N}-\frac{\pi a_{2} d^{6} Y^{2}}{4\left(\pi D^{2} N\right)^{3} D}+\frac{9 \pi a_{2}^{2} d^{8} Y^{4}}{64 D a_{1}\left(\pi D^{2} N\right)^{5}\left(A_{f}-M_{f}\right)} \\
& =K_{1}+K_{2} Y^{2}+K_{3} Y^{4}
\end{aligned}
$$

where $K_{1}=\left(a_{1}\left(T-M_{f}\right) d^{4} / 8 D^{3} N\right), \quad K_{2}=-\left(\pi a_{2} d^{6} Y^{2} / 4\right.$ $\left.\left(\pi D^{2} N\right)^{3} D\right)$, and $K_{3}=\left(9 \pi a_{2}^{2} d^{8} Y^{4} / 64 D a_{1}\left(\pi D^{2} N\right)^{5}\left(A_{f}-\right.\right.$ $\left.M_{f}\right)$ ).

Obviously, the stiffness of the SMA spring is related to the temperature, which will lay a foundation for the adjustment of the smooth parameter.

2.3. SMA-SD Oscillator System. The SMA-SD oscillator is established as shown in Figure 1, where the springs made of SMA can vary with the temperature.

The dynamic equation of the SMA-SD oscillator model is as follows:

$$
m \ddot{X}+2 K X\left(1-\frac{l}{\sqrt{X^{2}+l_{00}^{2}}}\right)=0
$$

where $m, X, K, l$, and $l_{00}$ are the quality of the mass block, the displacement of the mass block, the stiffness of the SMA spring ( $K$ in equation (14)), the length of the spring ( $l$ in equation (7)), and the horizontal distance from the mass block and the support, respectively. From equation (15), the stiffness of the SMA spring $K$ consists of three components and is a temperature-dependent variable.

Let $\alpha=\left(l_{00} / l\right), \quad x=(X / l)$, and $\omega_{0}^{2}=(2 K / m)$, and equation (15) can be written as

$$
\ddot{x}+\omega_{0}^{2} x\left(1-\frac{1}{\sqrt{x^{2}+\alpha^{2}}}\right)=0,
$$

where $\alpha=\left(l_{00} / l\right)=\left(l / l_{0}+\Delta \delta\right)=\left(l / l_{0}+\left(N \pi D^{2} / d\right)\left(1-\left(G_{M}\right.\right.\right.$ $\left.\left.\left(G_{T}\right)\right) \gamma_{\max }\right)$. Clearly, the smoothing parameter $\alpha$ can be changed depending on the temperature $T$, which means the natural frequency of the system can be changed by adjusting the temperature.

2.4. Restoring Force Analysis. The restoring force of springs in the SMA-SD oscillator is expressed as follows:

$$
F_{\mathrm{SD}}(x, T)=\frac{2 K}{m} x\left(1-\frac{1}{\sqrt{x^{2}+\alpha^{2}}}\right) .
$$

From equation (17), it can be seen that the restoring force is related to the displacement $x$ and the temperature $T$. At different temperatures, a graph of the relationship between the restoring force and displacement can be obtained as shown in Figure 2.

The parameters are chosen as $m=3 \mathrm{~kg}, d=0.002 \mathrm{~m}$, $D=0.03 \mathrm{~m}, M_{f}=22^{\circ} \mathrm{C}, N=10, M_{s}=48^{\circ} \mathrm{C}, A_{s}=50^{\circ} \mathrm{C}$, $a_{1}=9600, \quad a_{2}=12800, \quad G_{A}=15 \mathrm{GPa}, \quad G_{M}=6.5 \mathrm{GPa}$, $\gamma_{\max }=1.5 \%, L_{0}=0.05 \mathrm{~m}$, and $l=0.03 \mathrm{~m}$. In Figure 2, the restoring force curves are centrosymmetric with respect to the point $(0,0)$. When $T=25^{\circ} \mathrm{C}$, the restoring force curve is basically straight because the SMA just reaches the martensitic reverse temperature, resulting in little stiffness and almost no deformation. When $T=32^{\circ} \mathrm{C}$, instead of a monotonically increasing curve of the restoring force, there is a drop region, which indicates the presence of negative stiffness. When the temperature is $46^{\circ} \mathrm{C}$, the restoring force curve has the same trend as that at $T=32^{\circ} \mathrm{C}$, but the slope of the curve increases. For $T=58^{\circ} \mathrm{C}$, the peak and slope of the curve are greater than the previous temperatures. SMA transforms from low-temperature martensite to high-temperature austenite, and the spring stiffness increases significantly. At the same time, the spring also reaches a larger deformation.

2.5. Stiffness Analysis. From equation (17), the stiffness of the SMA-SD oscillator is given by

$$
\tilde{k}(x, T)=\frac{\partial F_{\mathrm{SD}}(x, T)}{\partial x} .
$$

The parameters are the same as above. The spring stiffness of the SMA-SD oscillator is analyzed.

From Figure 3(a), we can find that the value of stiffness is smallest and negative in the vicinity where the displacement is equal to zero, which indicates the existence of negative 


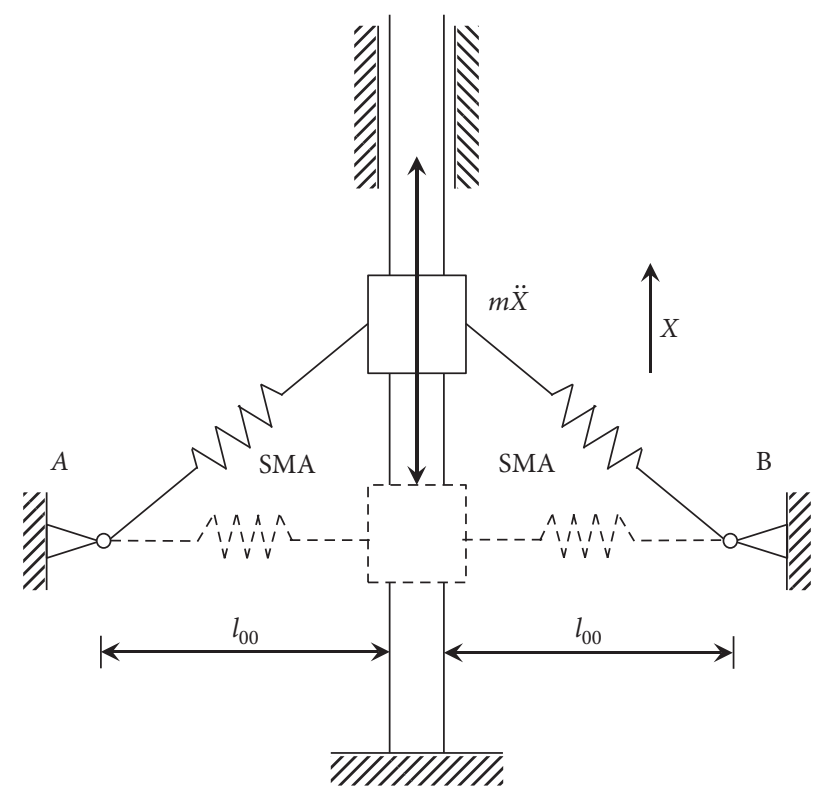

Figure 1: SMA-SD oscillator system model.

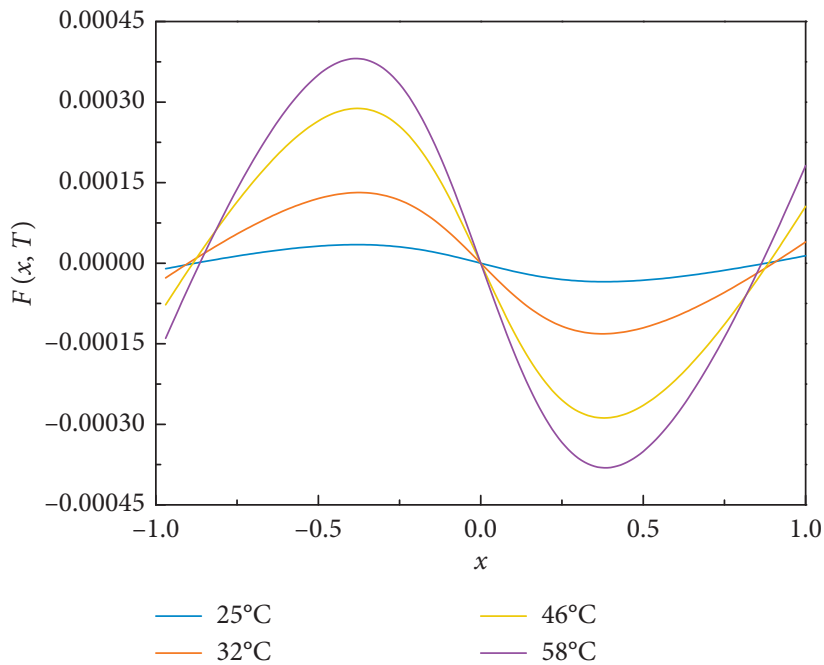

FIGURE 2: Restoring force-displacement of SMA-SD oscillator.

stiffness. It can be examined from Figure 3(b) that the entire surface is symmetrical about the axis $x=0$. In addition, with the increase of the temperature, the range of variation in stiffness becomes progressively larger.

Hence, in the SMA-SD oscillator system, the restoring force and stiffness exhibit nonlinear and changeful characteristics. The smooth parameters and stiffness associated with the natural frequency of the system are both related to the temperature.
2.6. The Relation between Temperature and Current. In order to adjust the SMA springs in the SD oscillator, the spring is heated by an electric current to control its temperature. The thermodynamic model is as follows [13]:

$$
\rho V C \frac{\mathrm{d} T}{\mathrm{~d} t}=P-h A\left(T-T_{\infty}\right),
$$

where $T_{\infty}, \rho, V, C, P, h$, and $A$ are the ambient temperature, the material density, the volume, the material specific heat, 


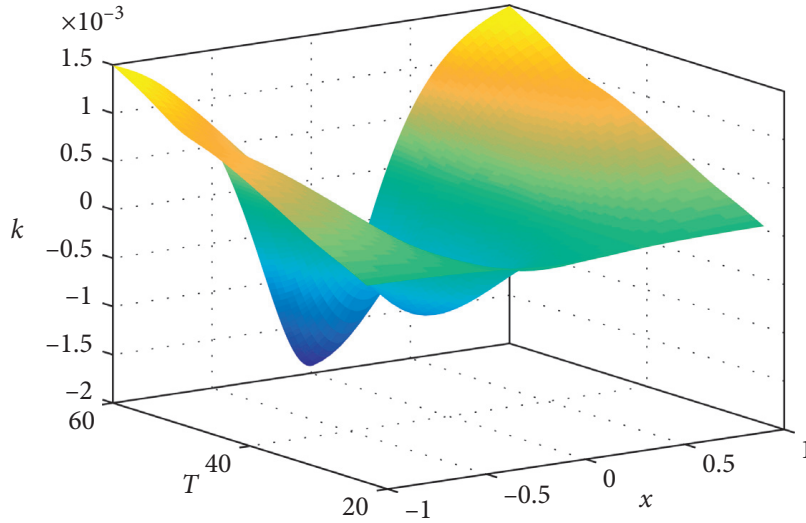

(a)

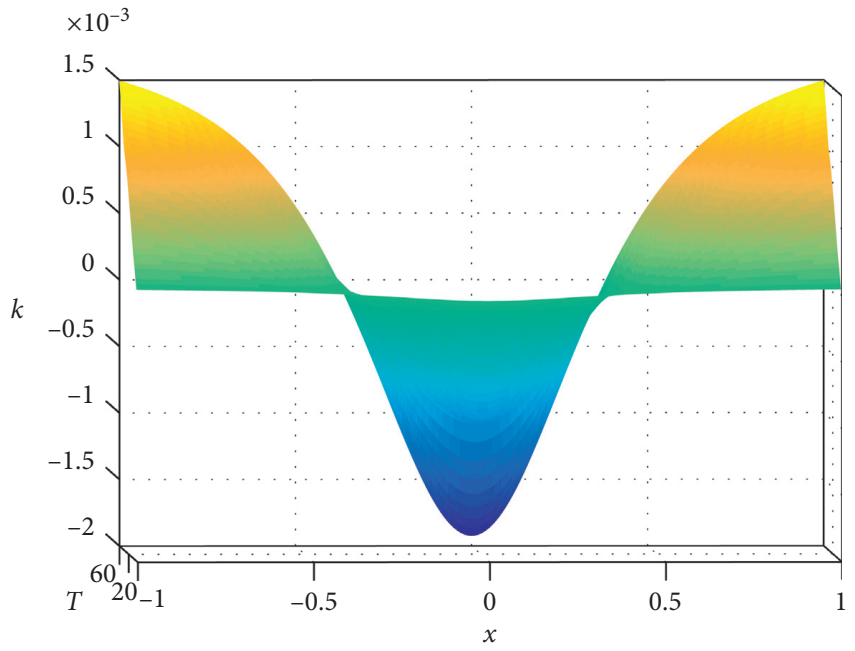

(b)

FIgURE 3: Stiffness of the SMA-SD oscillator.

the electric power when the current is heated, the convection coefficient, and the surface area of the component, respectively. Let $h$ be a constant. The electric power $P$ can be expressed as $P=I^{2} R$, where $I$ is the electric current and $R$ is the resistance.

Let $\widetilde{\tau}=(\rho V C / h A)$ and $c=(1 / h A)$, and the relationship between the temperature and the current is deduced as

$$
\tilde{\tau} \dot{T}+\left(T-T_{\infty}\right)=c I^{2} R .
$$

From equation (20), we know that the temperature can be regulated by adjusting the current intensity, which leads to realizing the frequency conversion for the SMA-SD oscillator.

\section{The Nonlinear DVA with Variable Frequency and Variable Damping}

Based on the frequency conversion characteristics of the SMA-SD oscillator, which can be controlled by the current intensity, a new model consisting of the SD oscillator with SMA springs and MRF is constructed. The schematic diagram of the dynamic vibration absorber designed is shown in Figure 4.

A mass is fixed on the piston rod. Two inclined springs made of SMA are connected to the mass at one end, and the other end is fixed on the shell of the absorber, thus forming the SMA-SD oscillator. The lower part of the absorber is a magnetorheological damper structure. There are wires in the piston rod. On the one hand, the wires wound on the springs are used to control the elongation and stiffness of the springs using the thermal effect of current. On the other hand, the magnetic field is generated by the magnetic effect of current to adjust the related characteristics of MRF. When the vibration absorber is working, the appropriate current according to the vibration signal is optimized to change the characteristics of the SMA springs and the MRF. Firstly, when SMA springs are electrified, they are heated up and deformed to a suitable length. And then the temperature remains constant, which completes the adjustment of the smooth parameters in the SD oscillator system. At this time, the stiffness of springs will also change due to the temperature. However, since the length and the stiffness of springs are both related to the temperature, a relatively moderate current should be selected based on the vibration signal. Meanwhile, the magnetic effect of the current is utilized to bring the viscosity of the MRF to the desired value. When the piston moves up and down, it receives both the restoring force produced by the SMA springs and the damping force. When the frequency of the designed DVA is the same as the excitation frequency, the resonance occurs and the vibration of the main system is suppressed.

For the designed absorber, variable stiffness can be achieved by winding coils on SMA springs. Specifically, by connecting an external power supply, the current intensity in the coils wounded on the SMA springs can be adjusted, thus changing the temperature of the SMA. Furthermore, we can see from Figures 2 and 3 that the designed DVA can obtain positive, negative, and zero stiffness under the temperature effect of SMA springs.

It is known that the damping force provided by MRF can be controlled by adjusting the current. Then, it can be expressed as [14]

$$
F_{D}=\left(b_{0} I^{2}+b_{1} I+b_{2}\right) \dot{y}=C \dot{y},
$$

where $b_{0}, b_{1}$, and $b_{2}$ are the parameters of MRF, which can be determined by experiments. $\dot{y}$ and $I$ are the equivalent damping of MRF and the current intensity.

From the above analysis, we can conclude that the designed nonlinear DVA consisting of the SMA-SD oscillator and MRF has not only the large energy dissipation and the adjustable damping but also the variable frequency. Furthermore, the frequency and damping of the absorber 


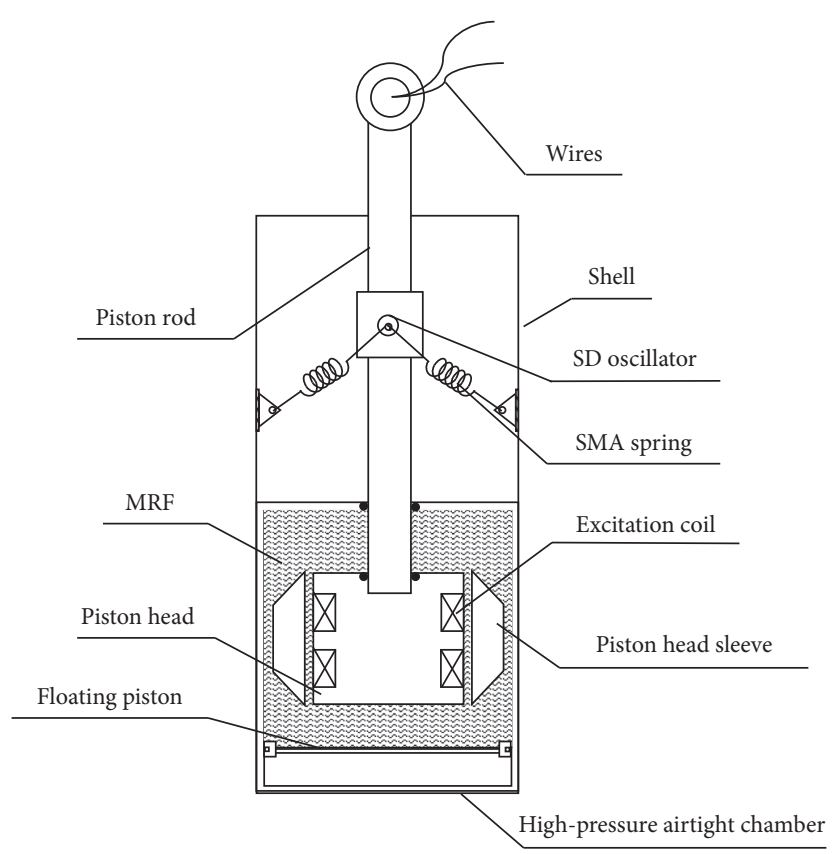

FIGURE 4: A sketch of the nonlinear dynamic vibration absorber.

can be adjusted by changing the current intensity, which is beneficial to practical engineering applications.

\section{The Application of the Designed Nonlinear DVA}

The loads on the beam bridge can take various forms, such as moving vehicle loads and wind loads. Based on this fact, the nonlinear DVA proposed in this paper can adjust its frequency or damping by changing the current intensity to adapt to different external environments. Consider the nonuniformed beam-vibration absorber model, as shown in Figure 5.

The variable cross-section beam bridge subjected to an infinite series of moving loads with speed $v$ is coupled with the designed absorber. The coupled system is mathematically modeled as

$$
\left\{\begin{array}{l}
\rho A_{0}(x) \frac{\partial^{2} u}{\partial t^{2}}+c_{0} \frac{\partial u}{\partial t}+\frac{\partial^{2}}{\partial x^{2}}\left(E I(x) \frac{\partial^{2} u}{\partial x^{2}}\right)=Q \delta(x-v t) \\
-F \delta\left(x-d_{\mathrm{DVA}}\right) \\
m \ddot{y}-F=0
\end{array}\right.
$$

where

$$
\begin{aligned}
F= & 2\left(K_{1}\left(u\left(d_{\mathrm{DVA}}, t\right)-y\right)-K_{2}\left(u\left(d_{\mathrm{DVA}}, t\right)-y\right)^{3}+K_{3}\left(u\left(d_{\mathrm{DVA}}, t\right)-y\right)^{5}\right) \\
& \cdot\left(1-\frac{l}{\sqrt{\left(u\left(d_{\mathrm{DVA}}, t\right)-y\right)^{2}+l_{00}}}\right)+k\left(u\left(d_{\mathrm{DVA}}, t\right)-y\right)+C\left(\frac{\partial\left(u\left(d_{\mathrm{DVA}}, t\right)-y\right)}{\partial t}-\dot{y}\right) .
\end{aligned}
$$

where $E$ is Young's modulus, $I$ is the second moment of area, $u(x, t)$ is the vertical displacement of the beam, $\rho A_{0}(x)$ is the mass of unit length, $L$ is the length of the beam, $c_{0}$ is the damping coefficient of the beam, $K_{1}, K_{2}$, and $K_{3}$ are the stiffness coefficient of the SMA spring, $k$ is the stiffness of $\mathrm{MRF}, C$ is the equivalent damping coefficient of the MRF, $d_{\text {DVA }}$ is the distance from the absorber to the beam bridge set, $Q$ is the equivalent mass of the load, and $v$ is the velocity of the moving load.
The boundary conditions of the variable-section beam are as follows:

$$
\begin{aligned}
& u(0, t) \frac{\partial^{2} u(0, t)}{\partial x^{2}}=0, \\
& u(L, t) \frac{\partial^{2} u(0, t)}{\partial x^{2}}=0 .
\end{aligned}
$$




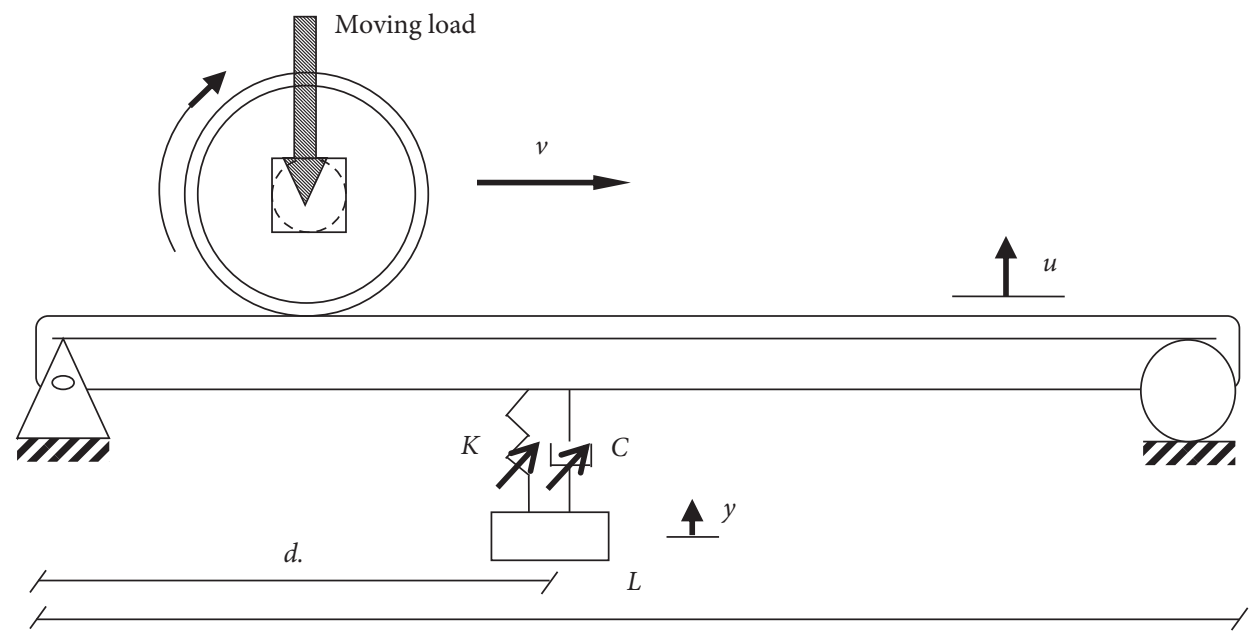

FIGURE 5: Nonuniformed beam-vibration absorber model.

The form of variable cross-section beam yields [15]

$$
\begin{aligned}
& h(x)=h_{0}\left(1-\beta \frac{x}{L}\right), \\
& A(x)=A_{0}\left(1-\beta \frac{x}{L}\right), \\
& I(x)=I_{0}\left(1-\beta \frac{x}{L}\right)^{3}, \\
& S(x)=\left(1-\beta \frac{x}{L}\right)^{3},
\end{aligned}
$$

where $h_{0}, A_{0}, I_{0}$, and $\beta$ are the height, the cross-sectional area, the inertia of the beam at $x=0$, and the variability of beam's cross section, respectively.

The first mode of the beam is the dominant mode in this application and a single-mode model can be adopted in this application. Then, $u$ will be written as

$$
u(x, t)=q(t) \sin \frac{\pi x}{L},
$$

where $q(t)$ is the amplitude of the mode.

Substituting equations (25) and (26) into (22), and by using the orthogonality of modes, integrating the first formula of equation (22) with respect to $x$ over $(0, L)$, we can obtain

$$
\left\{\begin{array}{l}
B_{1} \frac{\mathrm{d}^{2} q}{\mathrm{~d} t^{2}}+\frac{2 c_{0}}{\rho A_{0}} \frac{\mathrm{d} q}{\mathrm{~d} t}+\frac{E I_{0} \pi^{2}}{2 \rho A_{0} L^{4}} B_{2} q=\frac{4 Q}{\rho A_{0} L^{2}} \sin \frac{\pi v t}{L} \\
\frac{4 e}{\rho A_{0} L^{2}}\left\{k(e q-y)+C\left(e \frac{\mathrm{d} q}{\mathrm{~d} t}-\frac{\mathrm{d} y}{\mathrm{~d} t}\right)+2\left(K_{1}(e q-y)-\right.\right. \\
\left.\left.K_{2}(e q-y)^{3}+K_{3}(e q-y)^{5}\right)\left(1-\frac{l}{\sqrt{(e q-y)^{2}+l_{00}}}\right)\right\}, \quad 0 \leq t \leq \frac{L}{v} \\
m_{2} \frac{\mathrm{d}^{2} y}{\mathrm{~d} t^{2}}-\left\{k(e q-y)+C\left(e \frac{\mathrm{d} q}{\mathrm{~d} t}-\frac{\mathrm{d} y}{\mathrm{~d} t}\right)+2\left(K_{1}(e q-y)\right.\right. \\
\left.\left.-K_{2}(e q-y)^{3}+K_{3}(e q-y)^{5}\right)\left(1-\frac{l}{\sqrt{(e q-y)^{2}+l_{00}}}\right)\right\}=0
\end{array}\right.
$$

where $\quad e=\sin \left(\pi d_{\mathrm{DVA}} / L\right), \quad B_{1}=2-\beta, \quad$ and $B_{2}=(\beta-2)\left(\pi^{2} 2 \beta-2 \pi^{2}+3 \beta^{2}-\pi \beta^{2}\right)$. Here, the moving loads with the constant velocity at time $L / v$ intervals pass through the variable-section beam bridge. Further, to discuss the vibration of the beam subjected to the moving loads successively for a long time, we can use $|\sin \tau|(\tau \geq 0)$ to substitute $\sin \tau(0 \leq \tau \leq \pi)$ [16]. Hence, equation (30) yields 


$$
\left\{\begin{array}{l}
B_{1} \frac{\mathrm{d}^{2} q}{\mathrm{~d} t^{2}}+\frac{2 c_{0}}{\rho A_{0}} \frac{\mathrm{d} q}{\mathrm{~d} t}+\frac{E I_{0} \pi^{2}}{2 \rho A_{0} L^{4}} B_{2} q=\frac{4 Q}{\rho A_{0} L^{2}}\left|\sin \frac{\pi v t}{L}\right|- \\
\frac{4 e}{\rho A_{0} L^{2}}\left\{k(e q-y)+C\left(e \frac{\mathrm{d} q}{\mathrm{~d} t}-\frac{\mathrm{d} y}{\mathrm{~d} t}\right)+2\left(K_{1}(e q-y)-\right.\right. \\
\left.\left.K_{2}(e q-y)^{3}+K_{3}(e q-y)^{5}\right)\left(1-\frac{l}{\sqrt{(e q-y)^{2}+l_{00}}}\right)\right\} \\
m_{2} \frac{\mathrm{d}^{2} y}{\mathrm{~d} t^{2}}-\left\{k(e q-y)+C\left(e \frac{\mathrm{d} q}{\mathrm{~d} t}-\frac{\mathrm{d} y}{\mathrm{~d} t}\right)+2\left(K_{1}(e q-y)-\right.\right. \\
\left.\left.K_{2}(e q-y)^{3}+K_{3}(e q-y)^{5}\right)\left(1-\frac{l}{\sqrt{(e q-y)^{2}+l_{00}}}\right)\right\}=0 .
\end{array}\right.
$$

Let

$$
\begin{aligned}
\tau & =\omega_{1} t, \\
\bar{y} & =\frac{y}{l}, \\
\alpha & =\frac{l_{00}}{l}, \\
\bar{q} & =\frac{q}{l}, \\
\bar{p} & =e \bar{q}-\bar{y}, \\
\omega_{1}^{2} & =\frac{E I_{0} \pi^{2} B_{2}}{2 L^{4} \rho A_{0} B_{1}} .
\end{aligned}
$$

Equation (28) can be transformed into dimensionless equation as

$$
\left\{\begin{array}{l}
\frac{\mathrm{d}^{2} \bar{q}}{\mathrm{~d} t^{2}}+\bar{q}+\lambda_{1} \frac{\mathrm{d} \bar{q}}{\mathrm{~d} t}+2 \mu\left(\gamma_{11} \bar{p}-\gamma_{12} \bar{p}^{3}+\gamma_{13} \bar{p}^{5}\right)\left(1-\frac{1}{\sqrt{\bar{p}^{2}+\alpha^{2}}}\right) \\
+\mu \gamma_{2} \bar{p}+\mu \gamma_{3} \frac{\mathrm{d} \bar{p}}{\mathrm{~d} t}-f\left|\sin \omega_{1} \tau\right|=0 \\
\frac{\mathrm{d}^{2} \bar{p}}{\mathrm{~d} t^{2}}-e \frac{\mathrm{d}^{2} \bar{q}}{\mathrm{~d} t^{2}}+2\left(\gamma_{11} \bar{p}-\gamma_{12} \bar{p}^{3}+\gamma_{13} \bar{p}^{5}\right)\left(1-\frac{1}{\sqrt{\bar{p}^{2}+\alpha^{2}}}\right) \\
+\gamma_{2} \bar{p}+\gamma_{3} \frac{\mathrm{d} \bar{p}}{\mathrm{~d} t}=0,
\end{array}\right.
$$

where $\quad \omega=\left(\pi v / \omega_{1} L\right), \quad f=\left(4 Q / B_{1} L l \rho A_{0} \omega_{1}^{2}\right)$, $\mu=\left(4 e m_{2} / B_{1} L \rho A_{0}\right), \lambda=\left(2 c_{0} / B_{1} \rho A_{0} \omega_{1}\right), \gamma_{11}=\left(K_{1} / m_{2} \omega_{1}^{2}\right)$, $\gamma_{12}=\left(l^{2} K_{2} / m_{2} \omega_{1}^{2}\right), \gamma_{13}=\left(l^{4} K_{3} / m_{2} \omega_{1}^{2}\right), \gamma_{2}=\left(k / m_{2} \omega_{1}^{2}\right)$, and $\gamma_{3}=\left(C / m_{2} \omega_{1}\right)$.

To examine the effect of the designed nonlinear DVA, two sets of parameters of the nonuniformed beam and the moving load are chosen. In the two cases, the parameters of the nonlinear DVA have no change except the three currentcontrolled variables, i.e., the smooth parameters, the stiffness of the springs, and the viscosity of MRF. The first set of parameter values is listed in Table 1.

Here, the absorber is hung on the midspan of the beam, and the horizontal distance from the mass block and the support is $l_{00}=0.6 \mathrm{~m}$. Furthermore, the other parameter values of variable frequency and damping DVA are shown in Table 2.

Choose the heating temperature in SMA springs as $T=$ $46^{\circ} \mathrm{C}$ and the current intensity in $\mathrm{MRF}$ as $I=0.3 \mathrm{~A}$. Let $K_{1}=1997.438 \mathrm{~N} / \mathrm{m}, C=1500.170 \mathrm{~N} /(\mathrm{m} / \mathrm{s})$, and $\alpha=0.397$. Besides, the proposed DVA is abbreviated as VFDDVA. From Figure 6, we can see that the midspan amplitude of the beam bridge with vibration absorber decreases obviously.

The second set of parameter values of beam and load are listed in Table 3.

In the second case, for the designed DVA, only the parameters affected by current are changed, including the smooth parameters, the stiffness of the SMA springs, and the viscosity coefficient of MRF. The heating temperature in SMA spring and the current intensity are set as $T=55^{\circ} \mathrm{C}$ and $I=0.5 \mathrm{~A}$. Accordingly, the variable parameters are $K_{1}=2746.477 \mathrm{~N} / \mathrm{m}$, $C=3080.130 \mathrm{~N} /(\mathrm{m} / \mathrm{s})$, and $\alpha=0.352$. The second case of bridge mid-span vibration is shown in Figure 7. Similarly, the amplitude of the bridge with the designed absorber also decreases dramatically. From the above analysis, we can conclude that the designed nonlinear DVA can cope with different situations by changing the current intensity, which demonstrates the variable frequency and variable damping capabilities of the DVA.

Figure 8 shows the amplitude-frequency curve of the beam, where the vertical coordinate is the root mean square value of the acceleration of the beam after dimensionless. For the bare beam, a main peak near $\omega_{1}=1$ exists (see the black dotted line). Based on the VFDDVA, using the particle swarm optimization (PSO) algorithm for resonance condition, the peak for the beam is split into two new peaks. Clearly, the beam with the VFDDVA behaves better than the bare beam. Furthermore, the optimal method mentioned by Den Hartog [17] is applied in the design of the parameters for the VFDDVA. It can be seen that, with this optimization scheme, the damping frequency band is the widest. The results prove that the VFDDVA can achieve a better damping effect in a wide frequency domain by adjusting the parameters, which illustrates its potential for suppressing broad band vibration. 
TABle 1: The first set of values of beam and load.

\begin{tabular}{lcccccccc}
\hline$E_{0}(\mathrm{GPa})$ & $\rho_{0}\left(\mathrm{~kg} \cdot \mathrm{m}^{-3}\right)$ & $A_{0}\left(\mathrm{~m}^{2}\right)$ & $I_{0}\left(\mathrm{~m}^{4}\right)$ & $L(\mathrm{~m})$ & $\beta$ & $c_{0}\left(\mathrm{~N} \cdot \mathrm{s} \cdot \mathrm{m}^{-1}\right)$ & $Q(\mathrm{kN})$ & $v\left(\mathrm{~m} \cdot \mathrm{s}^{-1}\right)$ \\
\hline 11.591 & 7850 & 4.8 & 1.153 & 40 & 0.8 & 0.001 & 210 & 13 \\
\hline
\end{tabular}

TABLe 2: The first set of values of the designed DVA.

\begin{tabular}{ccccccccccccc}
\hline$M_{f}\left({ }^{\circ} \mathrm{C}\right)$ & $M_{s}\left({ }^{\circ} \mathrm{C}\right)$ & $A_{f}\left({ }^{\circ} \mathrm{C}\right)$ & $A_{s}\left({ }^{\circ} \mathrm{C}\right)$ & $G_{M}(\mathrm{GPa})$ & $G_{A}(\mathrm{GPa})$ & $\gamma_{\max }(\%)$ & $N$ & $D(\mathrm{~m})$ & $d(\mathrm{~m})$ & $l_{0}(\mathrm{~m})$ & $a_{1}$ & $a_{2}$ \\
\hline 22 & 45 & 60 & 50 & 5.5 & 7.5 & 2.7 & 30 & 0.9 & 0.45 & 1 & $3.55 \times 10^{5}$ & $7.13 \times 10^{7}$ \\
\hline
\end{tabular}

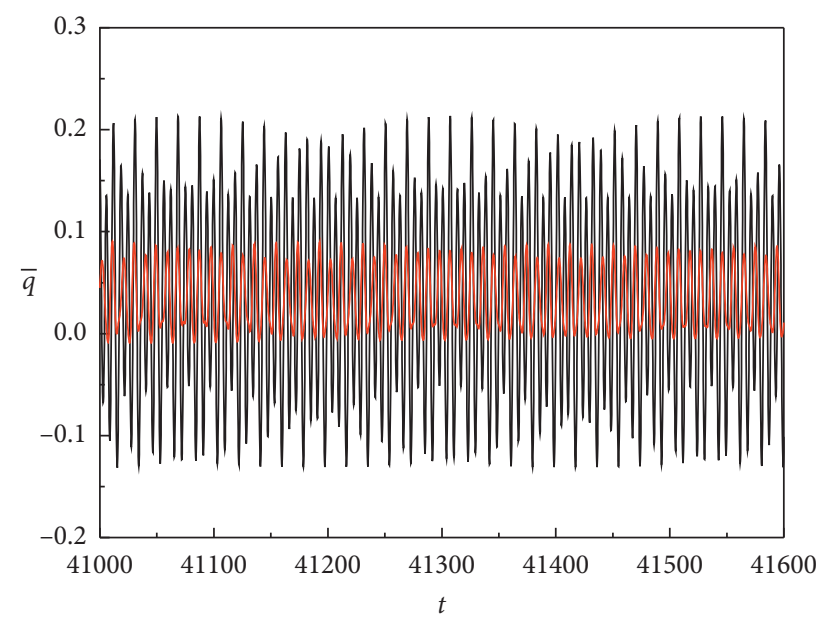

- Bare beam

— With VFDDVA

FIgURE 6: First maximum beam deflection.

TABLE 3: The second set of values of beam and load.

\begin{tabular}{lcccccccc}
\hline$E_{0}(\mathrm{GPa})$ & $\rho_{0}\left(\mathrm{~kg} \cdot \mathrm{m}^{-3}\right)$ & $A_{0}\left(\mathrm{~m}^{2}\right)$ & $I_{0}\left(\mathrm{~m}^{4}\right)$ & $L(\mathrm{~m})$ & $\beta$ & $c_{0}\left(\mathrm{~N} \cdot \mathrm{s} \cdot \mathrm{m}^{-1}\right)$ & $Q(\mathrm{kN})$ & $v\left(\mathrm{~m} \cdot \mathrm{s}^{-1}\right)$ \\
\hline 35.910 & 2500 & 19.274 & 1.043 & 60 & 0.1 & 0.001 & 189 & 75 \\
\hline
\end{tabular}

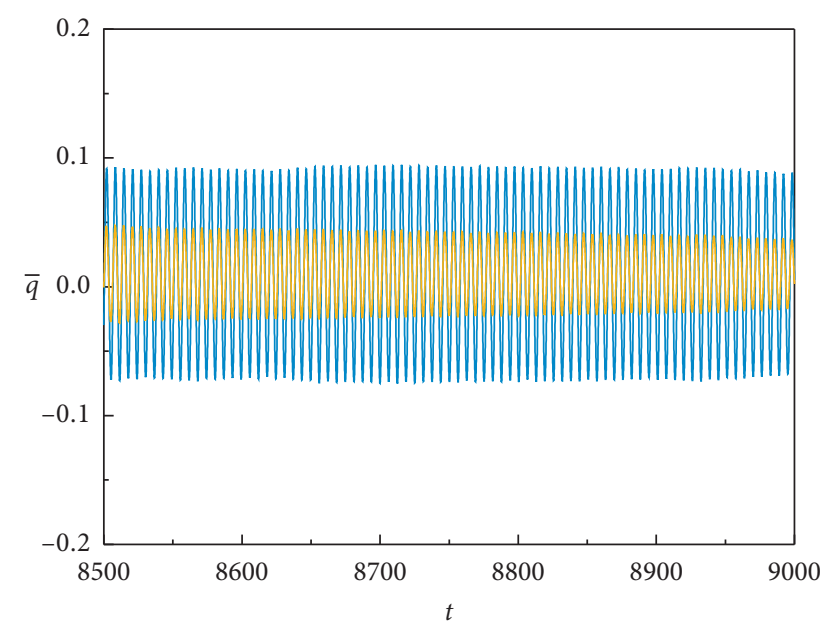

— Bare beam

- With VFDDVA

Figure 7: Second maximum beam deflection.

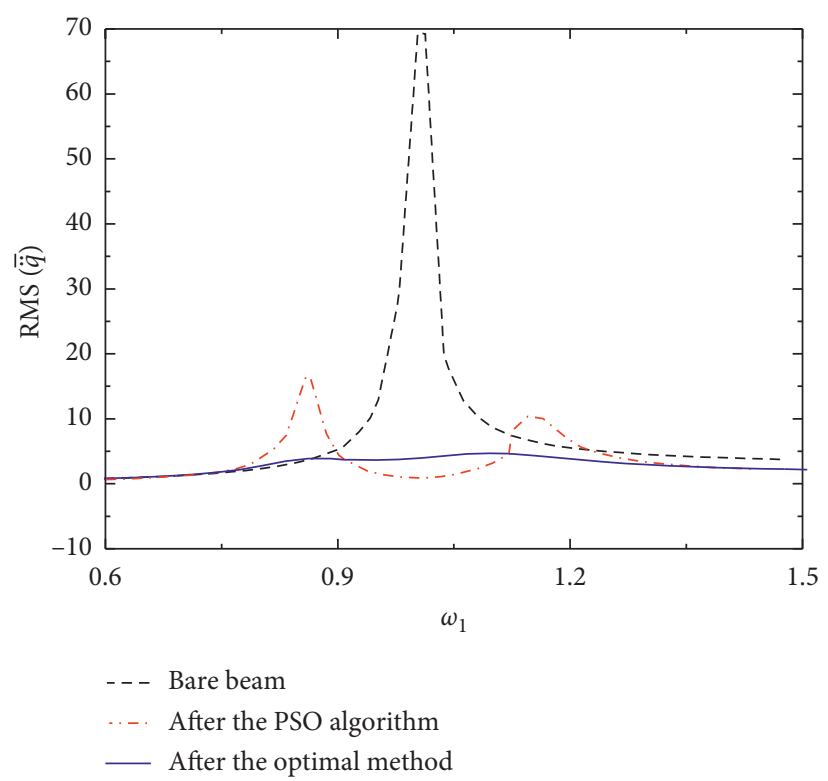

FIGURE 8: The amplitude-frequency curve of the beam. 


\section{Conclusion}

In this paper, SMA was introduced into the SD oscillator to adjust the smooth parameter $\alpha$. When the SMA springs transformed from martensite to austenite phase, the SMASD oscillator possessed the variable and controllable smoothness parameter $\alpha$, which was related to the natural frequency. By analyzing the effect of temperature on the stiffness and restoring force, it can be concluded that the SMA-SD oscillator can be a negative stiffness, zero stiffness, and positive stiffness system. The relationship between temperature and current proved that the SMA-SD oscillator can achieve frequency conversion by adjusting the current intensity. Furthermore, combining the SMA-SD oscillator and MRF, a nonlinear vibration absorber with variable frequency and damping was constructed. The current can be used to control the damping of the absorber according to the relationship between the damping force and the current. These results indicated that the designed VFDDVA can be managed actively to meet the changeable engineering environments by controlling the current intensity. The variable cross-sectional beam coupled with the designed VFDDVA was constructed to study the vibration attenuation and adjustability of the frequency and the damping of the absorber. The numerical results showed that the proposed VFDDVA can adapt itself to reduce the vibration energy of the beam in different conditions and achieve broadband vibration reduction by adjusting the current intensity, which promoted the application of the designed VFDDVA in engineering practice.

\section{Data Availability}

The data used to support the findings of this study are available from the corresponding author upon request.

\section{Conflicts of Interest}

The authors declare that there are no conflicts of interest regarding the publication of this paper.

\section{Acknowledgments}

This work was supported by the National Natural Science Foundation of China (nos. 12072203, 11872253, 11602151, and 11972237), the Hundred Excellent Innovative Talents in Hebei Province (no. SLRC2019037), the "333 talent project" in Hebei (no. A202005007), the Natural Science Foundation in Hebei Province of China (nos. A2019421005, A2019402043, and E2019210278), the Hebei Provincial Department of Education Project (nos. ZD2020328, QN2019149, and QN2018237).

\section{References}

[1] Q. J. Cao, M. Wiercigroch, and E. Pavlovskaia, “A structure for deoxyribose nucleic acid,” Physical Review E, vol. 74, Article ID 046218, 2006.

[2] R. L. Tian, X. W. Yang, Q. Zhang et al., "Vibration reduction in beam bridge under moving loads using nonlinear smooth and discontinuous oscillator," Advances in Mechanical Engineering, vol. 8, pp. 1-12, 2016.

[3] X. W. Yang, T. Wan, and R. L. Tian, "Vibration reduction of vehicle-bridge-coupled system using smooth-and-discontinuous absorber," Advances in Mechanical Engineering, vol. 10, pp. 1-11, 2018.

[4] C. Liang and C. A. Rogers, "Design of shape memory alloy springs with applications in vibration control," Journal of Vibration and Acoustics, vol. 115, no. 1, pp. 129-135, 1993.

[5] H. Ding, Z.-Q. Lu, and L.-Q. Chen, "Nonlinear isolation of transverse vibration of pre-pressure beams," Journal of Sound and Vibration, vol. 442, pp. 738-751, 2019.

[6] Z. J. Wu, X. J. Jing, J. Bian et al., "Vibration isolation by exploring bio-inspired structural nonlinearity," Bioinspiration. Biomimetics, vol. 10, Article ID 056015, 2015.

[7] R. Jacob, "The magnetic fluid cluth," Transactions of the American Institute of Electrical Engineers, vol. 67, pp. 13081315, 1948.

[8] R. G. Budynas and J. K. Nisbeet, Shigley's Mechanical Engineering Design, McGraw-Hill, New York, NY, USA, 9th edition, 2009.

[9] S. Degeratu, P. Rotaru, G. Manolea, H. O. Manolea, and A. Rotaru, "Thermal characteristics of Ni-Ti SMA (shape memory alloy) actuators," Journal of Thermal Analysis and Calorimetry, vol. 97, no. 2, pp. 695-700, 2009.

[10] T. E. Dye, "An experimental investigation of the behavior of nitinol," MS Thesis, Virginia Polytechnic Institute and State University, Blacksburg, VA, USA, 1990.

[11] F. Falk, "Model free energy, mechanics, and thermodynamics of shape memory alloys," Acta Metallurgica, vol. 28, no. 12, pp. 1773-1780, 1980.

[12] L. Duaval, M. N. Noori, Z. Hou et al., "Random vibration studies of an SDOF system with shape memory restoring force," Physica B, vol. 275, pp. 138-141, 2000.

[13] M. C. Piedboeuf, R. Gauvin, and M. Thomas, "Damping behaviour of shape memory alloys: strain amplitude, frequency and temperature effects," Journal of Sound and Vibration, vol. 214, no. 5, pp. 885-901, 1998.

[14] X. Y. Wang, X. Z. Zhou, and W. H. Li, "Variable stiffness and damping MR isolation system," in Proceedings of the 15th International Congress on Sound and Vibration, Daejeon, Korea, July 2008.

[15] S. M. Abdelghany, K. M. Ewis, A. A. Mahmoud, and M. M. Nassar, "Dynamic response of non-uniform beam subjected to moving load and resting on non-linear viscoelastic foundation," Beni-Suef University Journal of Basic and Applied Sciences, vol. 4, no. 3, pp. 192-199, 2015.

[16] R. L. Tian, X. W. Yang, Q. J. Cao et al., "The study on the midspan deflection of a beam bridge under moving loads based on SD oscillator," International Journal of Bifurcation and Chaos, vol. 22, Article ID 1250108, 2012.

[17] J. P. Den Hartog, Mechanica Vibrations, McGraw-Hill, New York, NY, USA, 1985. 